\title{
Radiological changes on chest CT following COVID-19 infection
}

\section{Dear Editor,}

COVID-19 infection is associated with high rates of hospitalisation and mortality, placing healthcare systems under strain. There are many reports regarding the non-contrast-enhanced high-resolution computed tomography (HRCT) features of the lungs during the onset of COVID-19; however, few studies have described the radiological changes and outcome of residual lesions in the lungs of recovered patients. ${ }^{1}$ Here, we review the HRCT features of recovered COVID-19 patients at 14-231 days post-discharge. These features can be utilised to predict prognosis and guide rehabilitation treatment of COVID-19.

A total of 56 cases diagnosed as COVID-19 between December 2019 and August 2020 were evaluated at our hospital.

Clinical data were collected from 56 recovered COVID-19 patients who were affected between December 2019 and August 2020. Among these patients, there were 38 mild and 18 severe cases based on the World Health Organization guidelines. ${ }^{2}$ The patients were re-examined by CT at 14-231 days post-discharge.

Clinical follow-up. At the first and second re-examination, the cardinal symptoms were chest tightness in 19 cases, shortness of breath in 16 cases, fatigue in 4 cases, and joint pain in 1 case. At the third and subsequent re-examinations, as the radiological changes improved, only 5 patients still had intermittent chest tightness.

CT features at the first re-examination. Among the 56 patients, HRCT revealed normal lungs in 17 $(30.36 \%)$ recovered patients and residual lung lesions in $39(69.64 \%)$.

Ground-glass opacities were found in 25 patients (44.64\%), with 1 showing a diffuse distribution of lesions in both lungs, 1 with a lesion in a single lung lobe, and $23(41.07 \%)$ with lesions in multiple lobular segments. The lesions were found in more than 2 lung lobes or segments, usually with non-uniform density, with more lesions in the lower lobes in both lungs. The lesions typically had an arc-shaped distribution in the peripheral lung field adjacent to the subpleural region.
Pulmonary interstitial shadows and fibrous stripes were found in 19 patients (33.93\%), including 5 who also had ground-glass opacities. Two cases (3.57\%) had interlobular septal thickening, presenting as pulmonary interstitial or interlobular septal thickening adjacent to the pleura; $2(3.57 \%)$ had subpleural linear shadows, presenting as an arched shadow parallel to the pleura within $1 \mathrm{~cm}$ of the subpleural region; 2 (3.57\%) had capillary bronchiectasis; and 13 (23.21\%) had irregular fibrous stripes (reticular changes), presenting as linear hyperdensities of varying length and thickness, which were generally found in the peripheral lung field and adjacent to the diaphragm. In the present study, we found no presence of a "crazy paving" pattern.

CT features at the second re-examination. A total of 39 recovered patients returned to the hospital for a second re-examination at 43-88 days post-discharge, 8 of whom had normal CT features of the lungs. All of the ground-glass opacities showed absorption to varying degrees, and were well absorbed in 15 patients, with a continued decrease in the extent of opacification and shrinkage of the lesions. Residual interstitial shadows were still present in 16 patients, where the irregular fibrous stripes, reticular changes, interlobular septal thickening, and subpleural linear shadows had not significantly improved.

CT features at the third or subsequent CT examinations. A total of 31 recovered patients returned to the hospital for a third or subsequent re-examination at 112-231 days post-discharge, 21 of whom had normal CT features of the lungs. All of the groundglass opacities showed substantial absorption: residual ground-glass opacities were found in 1 case, irregular fibrous stripes and reticular changes in 5 cases, interlobular septal thickening in 2 cases, and subpleural linear shadows in 1 case. The forced expiratory volume in 1 second (FEV1) percentage values for the 2 groups were different at the 3 re-examinations. The pulmonary function in the mild group was better than that in the severe group, and recovery in the mild group was faster (Table 1).

The persistent symptoms in patients after acute COVID-19 are of great concern. Re-examination by CT scan is necessary to assess pulmonary lesions in these patients. The lung function of a small number 


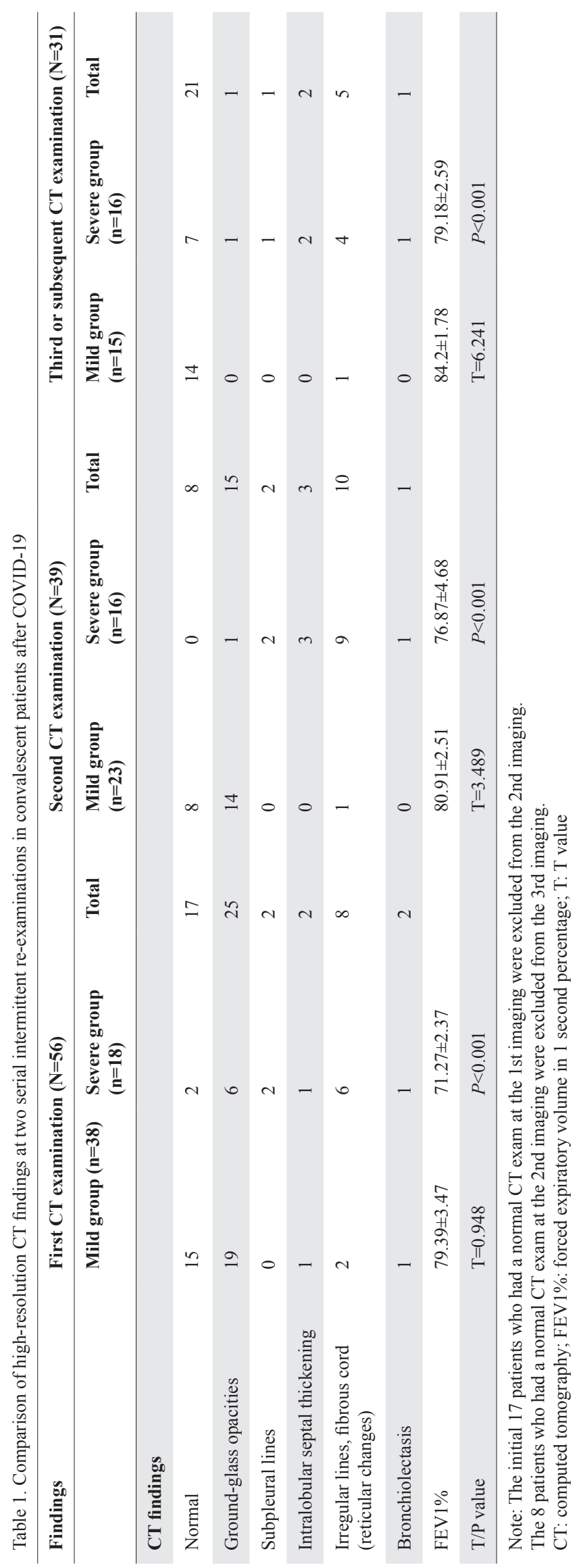

of COVID-19 patients has been shown to be severely impaired. The CT manifestations of these recovered COVID-19 patients are closely related to their activities of daily living. ${ }^{3,4}$

The residual lesions found by pulmonary CT scan in the recovered COVID-19 patients typically include ground-glass opacities and interstitial shadows (interlobular septal thickening, subpleural linear shadow, irregular stripe shadows, and reticular changes). Interstitial shadows do not occur alone following ground-glass opacities. In the present study, the ground-glass opacities were gradually absorbed and reduced in density, which was especially true when the lesions affected less than 2 lobes or were located in less than 2 pulmonary segments and had a lower density. The fibrous stripe shadows and subpleural linear shadows can also be partially absorbed; however, absorption is less likely for stripe shadows with a higher density. In some patients, the lesions remain almost unchanged on repeat CT scan 3 months later. Antonio et al. reported that stripe shadows in the pulmonary parenchyma, an irregular interface, and traction bronchiectasis are signs of fibrosis. ${ }^{5}$ These in turn are closely related to patient age and gender, with residual intrapulmonary fibrotic lesions more likely to be found in elderly men. According to autopsies of COVID-19 patients, the early lesions include exfoliation of bronchiolar epithelium, cilia shedding, squamous metaplasia, and atypically enlarged alveolar cells. ${ }^{6}$ At the early stage of COVID-19, some patients with fever do not present with apparent exudative lesions on chest X-ray or CT; the pulmonary shadows do not occur until 3-7 days later. This feature is in accordance with the fact that early pathological lesions primarily occur in the bronchi without abnormal findings in the lungs. COVID-19 infection causes stimulation to the epithelial cells, leading to intrapulmonary cell proliferation and squamous metaplasia. Patients with a course of disease shorter than 10 days have hyaline membrane formation in the lungs, alveolar cell proliferation, and oedema; those with a longer course of disease present with diffuse alveolar damage. ${ }^{7}$ Nicholls further divided the lesions into exudative, proliferative, and fibrotic stages. ${ }^{8}$ Johkoh et al. suggested that the ground-glass opacities present during the exudative stage of infectious pneumonia reflect oedema in the alveolar septum and formation of the hyaline membrane in the alveolar wall. During the proliferative and fibrotic stages (15-30 days), alveolar and interstitial proliferation and fibrosis were observed, indicating that the pathological changes were in accordance with the natural follow-up radiological results. ${ }^{9}$ 
During the follow-up period of 14-231 days, we found that the pulmonary function of convalescent patients with COVID-19 was damaged to some extent after discharge, which was related to residual ground-glass opacity, reticular changes, and fibrosis on CT. Especially in severe patients with acute respiratory distress syndrome and older male patients during hospitalisation, pulmonary diffusion function decreased significantly. It has been reported that the convalescent lesions of COVID-19 mainly exist in the alveolar wall, affecting the gas exchange through the alveolar capillary membrane and leading to a decline in diffusion function. During the early stage of rehabilitation in our study, the lung function of $91.1 \%$ of the convalescent patients recovered with prolongation of the re-examination time. The diffusion function of only 5 patients did not fully recover to the normal level. At present, we continue to follow up the pulmonary function of all patients. Follow-up of chest CT after discharge shows that HRCT findings of COVID-19 rehabilitation patients are closely related to their clinical manifestations, laboratory examination and pulmonary function. Following a reduction in groundglass opacities, reticular changes, and fibre stripes on $\mathrm{CT}$, residual lung lesions and lung function can be gradually improved, but interstitial shadow absorption remains relatively slow. At the same time, we found that, given the rampant infectivity of the COVID-19 virus, the psychological pressure of rehabilitation patients is tremendous. Substantial improvement in CT changes also accelerates the recovery of patients' mental health and aids re-integration into a normal social life. ${ }^{10}$

Re-examination by HRCT may reveal groundglass opacities and other residual lesions in recovered COVID-19 patients. As time progresses, the intrapulmonary lesions gradually improve or even disappear; however, the pulmonary interstitial shadows, reticular changes, and fibrous stripes have been noted to be absorbed more slowly.

\section{Acknowledgements}

The authors thank Weiping Gu and Si Luo for assistance with translating references. The present study was funded by the Agency for Integrated Care, Red Cross, and Development Board and Ministry of Hubei Health. The authors would like to thank the caregivers for taking time to participate in the research study and generously sharing their experiences and medical information.

\section{REFERENCES}

1. Zhou JC, Hui TCH, Tan $\mathrm{CH}$, et al. Chest Radiography in Coronavirus Disease 2019 (COVID-19): Correlation with Clinical Course. Ann Acad Med Singap 2020;49:456-61.

2. Chew SY, Lee YS, Ghimiray D, Tan CK, Chua GSW. Characteristics and Outcomes of COVID-19 Patients with Respiratory Failure Admitted to a "Pandemic Ready" Intensive Care Unit - Lessons from Singapore. Ann Acad Med Singap 2020; 49: 434-48.

3. An P, Wood BJ, Li W, et al.Postpartum exacerbation of antenatal COVID-19 pneumonia in 3 women. CMAJ. 2020;192:E603-6.

4. Goh KJ, Choong MC, Cheong EH, et al. Rapid Progression to Acute Respiratory Distress Syndrome: Review of Current Understanding of Critical Illness from COVID-19 Infection. Ann Acad Med Singap 2020;49:108-18.

5. Antonio GE, Wong KT, Hui DS, et al. Thin-section CT in patients with severe acute respiratory syndrome following hospital discharge: preliminary experience. Radiology 2003;228:810-5.

6. $\mathrm{Xu} \mathrm{Z}$, Shi L, Wang Y, et al. Pathological findings of COVID-19 associated with acute respiratory distress syndrome. Lancet Respir Med 2020;8:420-2.

7. Bhandari S, Sharma S, Bhargava A, et al. Inflammatory Markers in COVID-19. Ann Acad Med Singap 2020;49:393-7.

8. Nicholls JM, Poon LL, Lee KC, et al. Lung pathology of fatal severe acute respiratory syndrome. Lancet 2003;361:1773-8.

9. Johkoh T, Müller NL, Taniguchi $\mathrm{H}$, et al. Acute interstitial pneumonia: thin-section CT findings in 36 patients. Radiology 1999;211:859-63.

10. Ai T, Yang Z, Hou H, et al. Correlation of Chest CT and RT-PCR Testing for Coronavirus Disease 2019 (COVID-19) in China: A Report of 1014 Cases. Radiology 2020;296:E32-40.

Peng An, ${ }^{* 1}{ }_{M D}$ (Radiology), Weiping $\underline{\mathrm{Gu}},{ }^{* 1}{ }_{M B B S}$ (Radiology),

Si Luo, ${ }^{4}{ }_{M D}$, Min Zhang, ${ }^{3}{ }_{M B B S}$ (Public Health),

Yong Wang, ${ }^{4} M B B S$ (ICU, Anesthesiology),

Qiong-Xia Li $\underline{1}{ }^{2} M B B S$ (nffectious Disease)

${ }^{1}$ Division of Radiology, Xiangyang No. 1 People's Hospital Affiliated to Hubei University of Medicine, China

${ }^{2}$ Division of Infectious diseases, Xiangyang No. 1 People's Hospital Affiliated to Hubei University of Medicine, China

${ }^{3}$ Division of public health, Xiangyang No. 1 People's Hospital Affiliated to Hubei University of Medicine, China

${ }^{4}$ Postgraduate Training Basement of Hubei University of Medicine, Jinzhou Medical University, Xiangyang, China

\section{* Joint first authors}

Correspondence: Dr Yong Wang/ Dr Qiong-Xia Li, Department of Radiology and Infectious diseases, Xiangyang No. 1 People's Hospital, 75 Jiefangxi Road, Fancheng District, Xiangyang City, Hubei Province, 441000 China.

Email:wangyxyyy@163.com / lixyyy2015@163.com. 